\title{
20 CONFIGURING OF SUPPLY CHAIN NETWORKS BASED ON CONSTRAINT SATISFACTION, GENETIC AND FUZZY GAME THEORETIC APPROACHES
}

\author{
Alexander V. Smirnov ${ }^{1}$, Leonid B. Sheremetov ${ }^{2,3}$, Nikolai Chilov ${ }^{1}$ \\ Jose Romero Cortes ${ }^{2}$ \\ ${ }^{1}$ St.Petersburg Institute for Informatics and Automation of the Russian Academy of Sciences \\ SPIIRAS, 39, $14^{\text {th }}$ Line, St.Petersburg, 199178, Russia \\ smir@.iias.spb.su \\ ${ }^{2}$ Computer Science Research Center of National Technical University (CIC-IPN), \\ Av. Juan de Dios Batiz esq. Othon de Mendizabal s/n Col. Nueva Industrial Vallejo, \\ México, D.F., C.P. 07738, Mexico \\ sher@cic.ipn.mx \\ ${ }^{3}$ Mexican Oil Institute,
} Av. Lazaro Cardenas, 152, Col.san Bartolo Atepehuacan, México, D.F., CP 07730 ,

\begin{abstract}
In this article, the problem of the supply chain network ( $S C N)$ generation as a dynamic, flexible and agile system with dynamic configurations is considered. The proposed method consists in the product and SCN configurations with resource allocation in multiagent environment. For this purpose an application of different techniques is examined. Object oriented constraint networks are used for solving SCN configuration and resource allocation tasks using constraint satisfaction methodology. The second approach permits to find sub-optimal solution applying the theory of games with fuzzy coalitions. FIPA compliant agent platform and a CASE tool for SCN development, modeling and simulation are used to provide the experiments. The above techniques are compared and the simulation results are discussed.
\end{abstract}

\section{INTRODUCTION}

Supply-chain network (SCN) is a philosophy for enterprise integration based on a society formed by autonomous agents by bonding together to solve a common 
This paper presents several solutions to the problem of configuration of a SCN considered as a problem of task allocation among groups of autonomous agents. These groups are called coalitions. Important properties of the techniques and algorithms solving the task allocation problem are: (i) avoiding central authority, (ii) a low computational complexity, and (iii) increasing both individual utility and the overall outcome of the system (Shehory, 1999). There are two different approaches to the coalition formation problem, the former is based on the optimisation and combinatorial methods and the later on the game-theoretic approach (Axelrod, 1997; Jennings, 2001; Mares, 2000).

The paper considers the case when each task is attached to a group of agents (better to say, they try to form a coalition to perform this task) because they cannot be performed by a single agent, a single agent cannot perform them efficiently or cannot meet system's constraints. Earlier, the approach of forming fuzzy coalitions with full involvement was considered, when each agent may be a member of only one coalition and each task can be fulfilled by only one agent (Romero \& Sheremetov, 2001). In this paper an approach when capacity constraints are added to the problem statement is presented. Techniques of the first type are based on the conventional optimization approaches. Object oriented constraint networks are used for solving SCN configuration and resource allocation tasks using constraint satisfaction methodology (Smirnov and Chilov, 1999). The second approach permits to find sub-optimal solution applying the theory of games with fuzzy coalitions. The above techniques are compared and the simulation results are discussed.

\section{A CASE STUDY DESCRIPTION}

For the demonstration purposes a rather simple example of an automobile SCN was considered. The case study deals with production of a hypothetic vehicle (a Car). The price of the car is $\$ 20,000$. The production process (fig. 1) consists of the following two phases: Component Production and Car Assembly. Component Production consists of three parallel operations: Body Production, Motor Production, and Transmission Production (Bokma, 2001).

Demand is represented by a uniform distribution around the linear trend:

$d_{t}=a+b \cdot t+\mu \cdot \sigma$, where

$t$ - time instant

$d$-demand $\left(d_{t}\right.$ corresponds to time interval $\left.[t-1, t]\right)$

$a$ - basis value $(a=100)$

$b-$ trend component ( 0 for demand without trend)

$\mu$-random noise uniformly distributed within $[0: 1]$

$\sigma$-distribution amplitude

For simplicity, an example without trend and with low noise $(\sigma=5)$ is considered. In this case the technique of Single Moving Average for the demand forecasting can be used: 


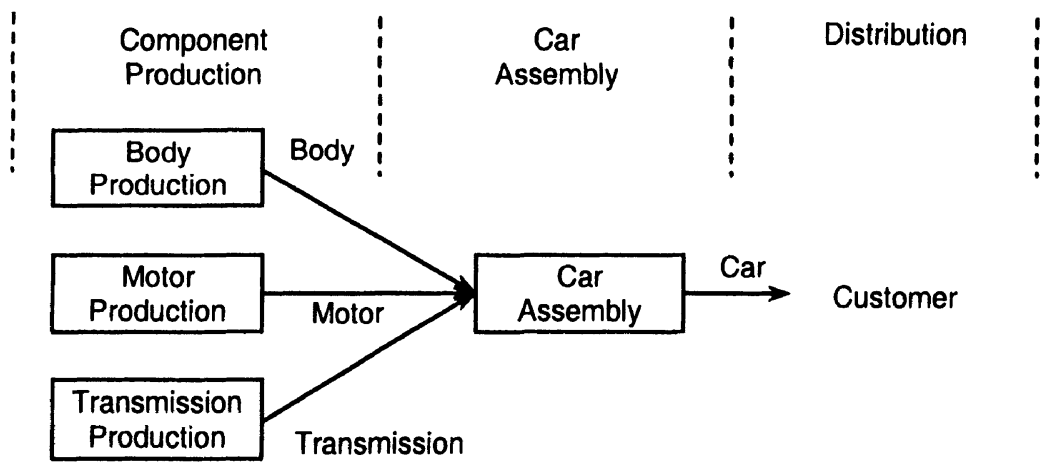

Figure 1 - Structure of the Production Process

$f_{t+2}=f_{t+1}=\frac{\sum_{i=t-n+1}^{t} d_{i}}{n}$, where

$f$ - forecasted data (forecast)

$n$ - forecast base $(n=5)$;

Car Assembly can be performed by one unit (car assembler or Unit 7) with the following parameters:

- Capacity = 105. A case without fixed capacity (no changes) is considered.

- Stocks are unlimited

- Stocking costs are $\$ 750$ per car/week, $\$ 300$ per body/week, $\$ 200$ per motor/week, $\$ 100$ per transmission/week.

- Assembly costs are $\$ 1500$ per car

Component Production can be performed by 6 units, each with different facilities (see Table ). The stocks are considered unlimited. Payoffs for Component Production are $\$ 7000$ per body, $\$ 5000$ per motor, and $\$ 4000$ per transmission. Penalties for backorders are $\$ 1500$ per car/week (paid by the car assembler to the customer), $\$ 400$ per body/week, $\$ 300$ per motor/week, $\$ 250$ per transmission/week (paid by the component producers to the car assembler).

Table 1. Data for the production units (potential supply chain members)

\begin{tabular}{|l|l|l|l|l|}
\hline Unit & Capacity & Facility & Production Costs & Stocking \\
\hline Unit 1 & 100 & Body Production & $\$ 4500$ per body & $\$ 250$ per body/week \\
\hline Unit 2 & 100 & Motor Production & $\$ 3500$ per body & $\$ 150$ per body/week \\
\hline Unit 3 & 100 & $\begin{array}{l}\text { Transmission } \\
\text { Production }\end{array}$ & $\begin{array}{l}\$ 2500 \text { per } \\
\text { transmission }\end{array}$ & $\begin{array}{l}\$ 50 \text { per } \\
\text { transmission/week }\end{array}$ \\
\hline Unit 4 & 303 & Body Production & $\$ 4900$ per body & $\$ 300$ per body/week \\
\hline & & Motor Production & $\$ 3800$ per body & $\$ 200$ per body/week \\
\hline & & $\begin{array}{l}\text { Transmission } \\
\text { Production }\end{array}$ & $\begin{array}{l}\$ 2700 \text { per } \\
\text { transmission }\end{array}$ & $\begin{array}{l}\$ 80 \text { per } \\
\text { transmission/week }\end{array}$ \\
\hline Unit 5 & 100 & Motor Production & $\$ 3600$ per body & $\$ 170$ per body/week \\
\hline & & $\begin{array}{l}\text { Transmission } \\
\text { Production }\end{array}$ & $\begin{array}{l}\$ 2600 \text { per } \\
\text { transmission }\end{array}$ & $\begin{array}{l}\$ 60 \text { per } \\
\text { transmission/week }\end{array}$ \\
\hline Unit 6 & 200 & Body Production & $\$ 4700$ per body & $\$ 270$ per body/week \\
\hline & & Motor Production & $\$ 3600$ per body & $\$ 170$ per body/week \\
\hline
\end{tabular}




\section{DESCRIPTION OF SOLUTION METHODS}

\subsection{Constraint Satisfaction Approach to Configuration and Resource Allocation of the SCN}

Generally a task of a resource optimization can be described as follows: it is necessary to define efficient allocation (where is situated) and schedule (when is available) of a given resource components and when and which of the components should be used. As a criterion such indices as costs of the resource acquiring and/or delivery or required for this purpose time, can be used. It is necessary to take into account relationships existing between resources and resource components (for instance, a resource may consist of some other resources). The fact of existence of these relationships allow considering the task of resource optimization as a constraint satisfaction problem (Smirnov and Chilov, 1999).

A constraint satisfaction problem is defined as $(X, D, C)$. It consists of $\mathrm{n}$ variables $X=\left\{x_{1}, x_{2}, \ldots, x_{n}\right\}$, whose values are taken from finite, discrete domains $D=\left\{D_{1}, D_{2}, \ldots, D_{n}\right\}$, and a set of constraints on their values $C=\left\{c_{1}, c_{2}, \ldots c_{m}\right\}$. A constraint is defined by a predicate. That is, the constraint $c_{k}\left(x_{k l}, \ldots, x_{k j}\right)$ is a predicate that is defined on the Cartesian product $D_{k 1} \times \ldots \times D_{k j}$. This predicate is true if the value assignment of these variables satisfies this constraint. Solving a constraint satisfaction problem assumes finding an assignment of values to all variables such that all constraints are satisfied. In other words, a constraint satisfaction problem is a task of finding a consistent assignment of values to variables (Mackworth, 1992; Yokoo, and Hirayama 2000). A simple example of such task is given in (fig. 2).

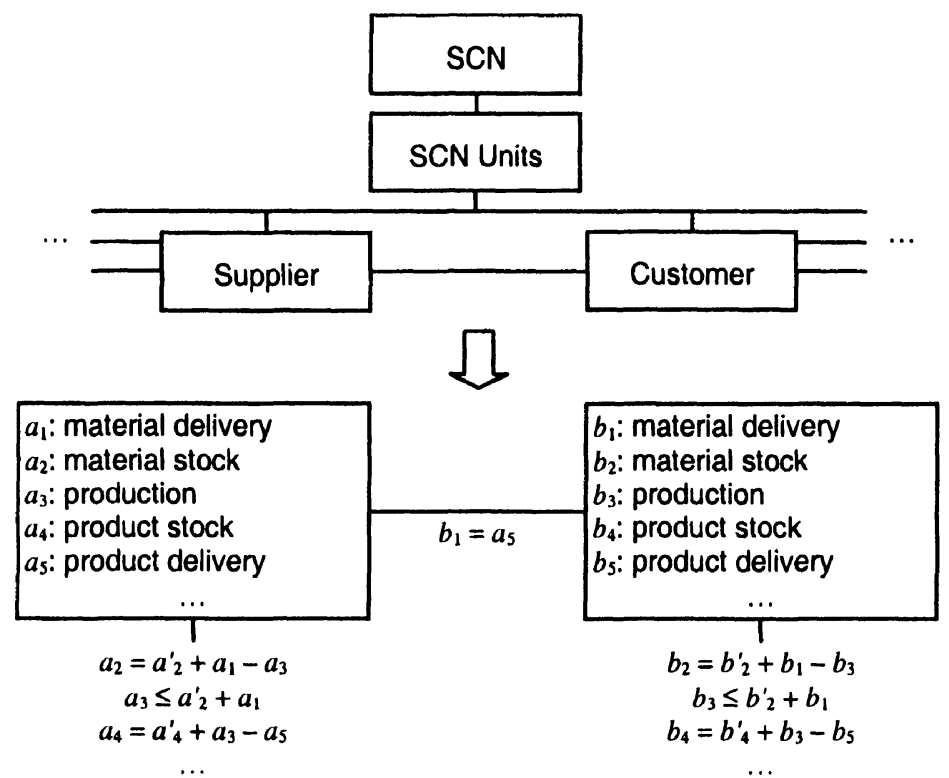

Figure 2 - Example constraint network

Regarding to the considered here task, the components of the task have the following notations: 
$x_{i j t}$ - the quantity of the $j$ component to be produced by unit $i$ at a time interval $[t-1, t]$;

$D_{i j t}$ the sets of integer positive numbers $\left(I^{+}\right)$;

$C$ - the constraints responsible for synchronization and consistency of the considered SCN system. The following groups of constraints were selected: capacity-based constraints, constraints on materials availability, delivery or synchronization constraints.

\subsection{Game Theoretic Approach with Fuzzy Coalitions to SCN Configuring}

Another approach developed in this article is based on fuzzy coalition game of players with full involvement (Mares, 2000; Romero and Sheremetov, 2002). Each player corresponds to the node of the SCN. According to (Mares, 2000), the core (the set of solutions) $C_{F}$ of the game $(I, w)$ (where $I$ is the set players and $w$ is the characteristic function associated with fuzzy payoffs) is defined as follows:

$$
C_{F}=\left\{\sum_{i \in I} x_{i} \leq w(I), \sum_{i \in K} x_{i} \leq w(K): K \subset I\right\}
$$

The core has the following membership function associated with it:

$$
\gamma_{c}(x)=\min _{K \subset I}\left(v_{\succ=}\left(w(I), \sum_{i \in I} x_{i}\right), v_{\prec=}\left(\sum_{i \in K} x_{i}, w(K)\right)\right)
$$

where: $v \succ=\left(w(I),\left\langle\sum_{i \in I} x_{i}\right\rangle\right)$, with the preference $\succ=$ as a weak order fuzzy relation with membership function $v \succ=: R \times R \rightarrow[0,1]$.

The expressions (1) and (2) correspond to the game-theoretic model with fuzzy coalitions and contain only the terms relative to the fuzzy payments. For the purposes of this article the fuzzy core was changed to include the capacities of each agent and the tasks to fulfill defined by the demand, product bill and specified in the problem domain ontology. The new core definition follows (without lacking of generality, for simplicity this model doesn't include the fluctuations caused by inventories):

$$
\begin{aligned}
& C=\left\{2500 x_{11 t}+2100 x_{41 t}+2300 x_{61 t}+1500 x_{22 t}+1200 x_{42 t}+1400 x_{52 t}+1400 x_{62 t}+\right. \\
& 1500 x_{33 t}+2500 x_{74 t}+1300 x_{43 t}+1400 x_{53 t} \geq(100+5 t+\mu 5) w(I), \\
& 2500 x_{11 t}+2100 x_{41 t}+2300 x_{61 t} \leq(100+5 t+\mu 5) w\left(k_{1}\right) \\
& 1500 x_{22 t}+1200 x_{42 t}+1400 x_{52 t}+1400 x_{62 t} \leq(100+5 t+\mu 5) w\left(k_{2}\right) \\
& 1500 x_{33 t}+1300 x_{43 t}+1400 x_{53 t} \leq(100+5 t+\mu 5) w\left(k_{3}\right) \\
& 2500 x_{74 t} \leq(100+5 t+\mu 5) w\left(k_{4}\right) \\
& x_{11 t}+x_{41 t}+x_{61 t}=100+5 t+5 \mu \\
& x_{22 t}+x_{42 t}+x_{52 t}+x_{62 t}=100+5 t+5 \mu \\
& x_{33 t}+x_{43 t}+x_{53 t}=100+5 t+5 \mu
\end{aligned}
$$




$$
\begin{aligned}
& x_{74 t}=100+5 t+5 \mu \\
& x_{11 t} \leq 100 \quad x_{41 t} \leq 302.5 \\
& x_{22 t} \leq 100 \quad x_{42 t} \leq 302.5 \\
& x_{33 t} \leq 100 \quad x_{43 t} \leq 302.5 \\
& x_{61 t} \leq 200 \\
& x_{52 t} \leq 100 \quad x_{62 t} \leq 200 \\
& x_{74 t} \leq 102.5 \\
& \left.x_{i j t} \in R^{+}, i=1, \ldots, 7 ; j=1, \ldots, 4 \quad t=1, \ldots, 5\right\} \\
& \text { where: } \\
& x_{i j t}=\text { the quantity of the } j \text { component to be produced by agent } i \text { in time } t \text {. } \\
& w(I)=\text { fuzzy payoff per unit for car production. } \\
& w\left(k_{1}\right)=\text { fuzzy payoff per unit for Body Production. } \\
& w\left(k_{2}\right)=\text { fuzzy payoff per unit for Motor Production. } \\
& w\left(k_{3}\right)=\text { fuzzy payoff per unit for Transmission Production. } \\
& w\left(k_{4}\right)=\text { fuzzy payoff per unit for car assembly. } \\
& \mu=\text { uniform random variable in }[0,1] \text {. } \\
& 100+5 t+5 \mu \text {, for } t=1, \ldots, 5 \text { and } \mu \text { is uniform in }[0,1]
\end{aligned}
$$

Two solution techniques were studied. First, the Excel solver was used. Next, genetic algorithms were used and Evolver software. Testbed implementation details and simulation results are discussed in the following section.

\section{IMPLEMENTATION DETAILS AND OBTAINED RESULTS}

\subsection{A Multiagent Testbed}

To implement the prototype and provide the experiments with the proposed methods, a multiagent testbed has been developed. For the implementation of the testbed, Zeus v1.2.1 software (JRE v1.3.1.02) was used as FIPA compliant agent platform (Nwana et all., 1999).

Each domain agent associated with a node of the SCN was equipped with the contract net negotiation protocol (CNP). The SCN Head agent (called Assembly in the presented case study) was the one who decided the final configuration based on the computations performed by the solvers. Problem solving methods (PSM) are implemented as external legacy software modules. Genetic algorithms are implemented using the Evolver software (Evolver, 2001), accessed through the Excel Wrapper agent. Constraint solver is implemented using ILOG Configurator constraint programming engine (ILOG, 2001) and accessed by means of another Wrapper agent implemented in $\mathrm{C}++$. Agents were running on the agent platform and PSM software on another computer.

Some scalability problems were detected while running the simulations. First, an execution of the batch files generated in a predetermined way: runl.bat (executing Agent Name Server - ANS), run2.bat (domain agents) and run3.bat (visualizer and Directory Facilitator - DF), was tried. The problem of this approach is that each 
agent is executed in his own virtual machine consuming many computer resources. As a result, it was impossible to execute more then 10 agents in a single-host fashion. To handle this problem, the utility (Zsh) that allows to execute several agents in the same virtual machine was used. The second parameter of this utility $<$ param $2>$ is a number representing the time period that any DF agent started will wait between querying the agent system for their capacities to be properly recognized by the rest of the system, which can affect the system performance. This way it was possible to reach the following configuration: 10 Tail Agents, Assembler Agent, ANS, Visualizer Agent, and DF Agent. The agents were running on a Compaq Presario 5473 (Celeron $550 \mathrm{Mhz}, 128 \mathrm{MB}$ RAM) computer. Using this computer, the case study SCN shown in the figure 3 has been simulated.

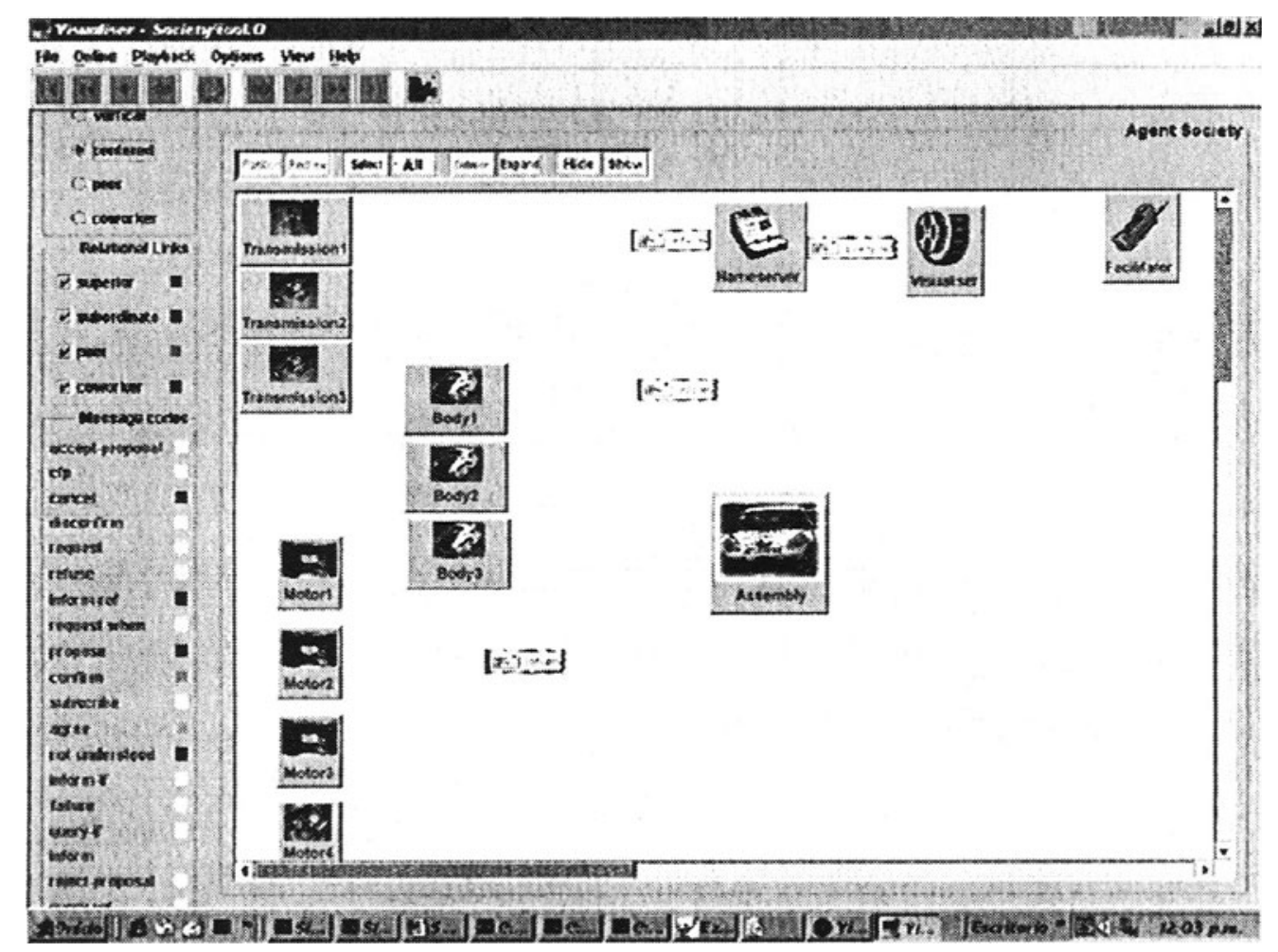

Figure 3 - A screenshot of the Visualizer with an example configuration (Society Viewer's window).

As it shown in fig.3, the case study consisted in eleven domain agents (one for each facility and an assembly one) and three utility agents of the platform. Each domain agent had an access to a DB where it's capacity, production cost and stocking data was stored. In the case of a fuzzy game, domain agents were also equipped with the interface windows to capture the parameters of the individual membership functions. Special purpose Coalition Agents have been generated for each component every time a new demand occurred. Additional software accessed by these agents has been developed to calculate coalition membership functions. Assembly Agent served as a SCN coordinator initiating negotiation protocols. CNP was used to collect the biddings for the demand. 


\subsection{Results of Constraint Satisfaction Approach}

As it was mentioned above to solve considered here task the engine of ILOG Configurator was used. It represents constraint network to be processed in an objectoriented form. The system supports the following two types of relations: (i) "is a" between classes and objects, and (ii) "is connected to" between objects. In turn relations "is connected to" can be of the following two subtypes: (i) "has part" or "part of" and (ii) "uses". The objects own attributes whose values belong to defined domains and are controlled by constraints existing in the problem domain. This representation allows rapid and convenient model creation and maintenance. On the other hand an advanced ILOG Solver performs the task solving process in an efficient way. Obtained results for 5 time intervals are given in table 2 .

Table 2. Production levels per unit for five time intervals

\begin{tabular}{|l|l|l|l|l|l|l|l|l|l|l|l|}
\hline$t$ & $x_{11 t}$ & $x_{22 t}$ & $x_{33 t}$ & $x_{41 t}$ & $x_{42 t}$ & $x_{43 t}$ & $x_{52 t}$ & $x_{53 t}$ & $x_{61 t}$ & $x_{62 t}$ & $x_{74 t}$ \\
\hline 1 & 100 & 100 & 100 & 0 & 0 & 0 & 0 & 5 & 5 & 5 & 105 \\
\hline 2 & 100 & 100 & 100 & 0 & 0 & 0 & 0 & 12 & 12 & 12 & 112 \\
\hline 3 & 100 & 100 & 100 & 0 & 0 & 0 & 0 & 15 & 15 & 15 & 115 \\
\hline 4 & 100 & 100 & 100 & 0 & 0 & 0 & 0 & 24 & 24 & 24 & 124 \\
\hline 5 & 100 & 100 & 100 & 0 & 0 & 0 & 0 & 28 & 28 & 28 & 128 \\
\hline
\end{tabular}

The common network payoffs per car obtained for each time interval are equal $9,480.95 ; 9,457.14 ; 9,447.83 ; 9,422.58 ; 9,412.50$ respectively. The payoffs $(p)$ of the participating units per car/component are as follows: $p_{\text {unit } 1}=2,500 ; p_{\text {unit } 2}=1,500$; $p_{\text {unit } 3}=1,500 ; p_{\text {unit } 4}=0 ; p_{\text {unit } 5}=1,400 ; p_{\text {unit } 6}=1,850 ; p_{\text {unit } 7}=2,500$.

Utilizing combination of constraint-based technique allowing obtaining a global optimal solution, and ILOG Solver engine allowing utilizing both linear and nonlinear constraints, makes it possible to solve large tasks with complicated dependencies between their components.

\subsection{Results of Game Theoretic Approach with Fuzzy Coalitions}

For the case study, positive ramp membership functions were selected: payoff per car - $[7,100,8,000]$; gross payoffs for body - $[6,500,7,000]$; motor - $[4,500,5,000]$; transmission - $[3,800,4,000]$; and assembly - $[2,000,4,000]$ respectively. Two solvers were used to find the solution. The first one, the Excel solver was able only to approximate the integer solution (table 3). Table 3 shows the imputations for each time interval, the last column shows the number of cars to be assembled according to the forecasting defined by (4).

Table 3. Production levels per unit for five time intervals

\begin{tabular}{|l|l|l|l|l|l|l|l|l|l|l|l|}
\hline$t$ & $x_{11 t}$ & $x_{22 t}$ & $x_{33 t}$ & $x_{41 t}$ & $x_{42 t}$ & $x_{43 t}$ & $x_{52 t}$ & $x_{53 t}$ & $x_{61 t}$ & $x_{62 t}$ & $x_{74 t}$ \\
\hline 1 & 100 & 100 & 100 & 0 & 0 & 0 & 5.299 & 5.3 & 5.3 & .001 & 105.3 \\
\hline 2 & 100 & 100 & 100 & 0 & 0 & 0 & 8.399 & 11.5 & 11.5 & 3.101 & 111.5 \\
\hline 3 & 100 & 100 & 100 & 0 & 0 & 0 & 10.25 & 15.2 & 15.2 & 4.95 & 115.2 \\
\hline 4 & 100 & 100 & 100 & 0 & 0 & 0 & 14.50 & 23.7 & 23.7 & 9.20 & 123.7 \\
\hline 5 & 100 & 100 & 100 & 0 & 0 & 0 & 16.75 & 28.2 & 28.2 & 11.45 & 128.2 \\
\hline
\end{tabular}


The second solution method used was that of genetic algorithms. The solution converged within several seconds (though communications between agents took considerable time). The rates of mutation and crossover in the typical values of 6 and $50 \%$ respectively, with a size of population of 50 organisms were fixed. Time convergence parameters and number of iterations was also fixed (Romero and Sheremetov, 2001).

The common network payoffs per car obtained for each time interval are equal to $7,578.46,7,578.22,7,578.22,7,577.84,7,577.84$ respectively. The payoffs $(p)$ of the participating units per car/component are as follows: $p_{\text {unit } 1}=2,500 ; p_{\text {unit } 2}=1,500$; $p_{\text {unit } 3}=1,500 ; p_{\text {unit } 4}=0 ; p_{\text {unit } 5}=1,400 ; p_{\text {unit } 6}=1,400 ; p_{\text {unit } 7}=2,500$. The same gross payoffs per unit were obtained for each time interval for each component: $w(I)=$ 20,$000 ; w\left(k_{1}\right)=7,000 ; w\left(k_{2}\right)=5,000 ; w\left(k_{3}\right)=4,000 w\left(k_{4}\right)=4,000$. The possibility of the fuzzy game $\gamma_{c}(I, w)=1.00$ (because of the simplicity of the case study), though the imputation obtained took into account the subjective estimations of the players defined by their fuzzy payments.

\section{DISCUSSION AND CONCLUSIONS}

Different problem statements and solution techniques have been discussed in this article. The problem statement defined in section 3.2 permits to consider non-lineal membership functions, integer variables, multi-objective functions and non-lineal constraints. But only the use of heuristic and soft-computing techniques such as genetic algorithms as it was shown in (Romero and Sheremetov, 2001), permit to find the best converged imputation in a reasonable time for real world applications. The use of genetic algorithms has the following benefits: they allow to find a semioptimal solution in the case when an optimal solution can't be found analytically because of the problem's complexity, they allow to find a local optimal solution in the cases when the optimal solution even doesn't exist (when the game is not convex), and also they can be used to find solutions for the mixed approaches similar to the discussed in this paper when a game-theoretic approach is combined with the combinatorial one.

The constraint-based approach assumes complete information sharing while the game theoretic approach assumes minimal or no information sharing. Since in realworld situations the both cases as well as some intermediate ones are possible, the application of presented here techniques in a combined way is preferable. The subsequent research efforts are planned to be devoted to investigation of combined techniques for tasks representing more realistic situations according to the following:

1) wider range of parameters to optimise,

2) more complicated strategies of the units (e.g., including safety stocks forming),

3) more complicated demand patterns and forecasting techniques,

4) non-lineal relationships in the model.

FIPA compliant agent platform and a CASE tool for SCN development, modeling and simulation are used to provide the experiments. The use of this platform not only ensures interoperability among the agents and reusable components for the future enterprise-based applications, but also allows taking 
advantage of agent-based, as well as component-based services offered by the SCN units that can be used and customized as needed.

The presented methodology of the coalition formation implies implicit negotiations (vague, imprecise, open), which are guided to maximize the benefits or viabilities (i) of each individual agent of the SCN, (ii) of agents' coalition and even (iii) of a great coalition that contains all the agents of the system. The structure of a virtual company formed this way, has the distinctive point and the advantage in that it takes into account the convenience for the companies to participate in groups (virtual companies), it allows quick adaptations that respond to changes of the environmental dynamics.

\section{ACKNOWLEDGMENTS}

Partial support for this research work has been provided by the CONACyT, Mexico within the project 31851-A Models and Tools for Agent Interaction in Cooperative MAS and by the National Technical University, Mexico, within the program CGEPI 18.01. The paper is also due to the research carried out as a part of the project \# 4.4 of the research program \# 18 "Intelligent Computer Systems" of the Russian Academy of Sciences, and grant \# 02-01-00284 of the Russian Foundation for Basic Research. Some examples were developed using software granted by ILOG Inc.

\section{REFERENCES}

1. Axelrod, R. (1997). The complexity of cooperation: agent based models of competition and collaboration. Princeton University Press.

2. Bokma, A. (2001). CogNet: Integrated Information and Knowledge Management and its Use in Virtual Organisations. In E-Business and Virtual Enterprises, Managing Business-to-Business Cooperation. L.M.Camarinha-Matos, H.Afsarmanesh, R.J.Rabelo, ed. Kluwer Academic Publishers, Boston, 361-370.

3. Evolver (2001). Release 4.01. Palisade.

4. Jennings, N.R., Faratin, P., Lomuscio, A.R., Parsons, S., Sierra, C and Wooldridge, M., (2001). Automated negotiation: prospects, methods and challenges. Int. J. of Group Decision and Negotiation 10(2): 199-215.

5. ILOG (2001). Corporate website, URL: http://www.ilog.com.

6. Mackworth, A. (1992). Constraint Satisfaction. In: S.C. Shapiro (ed.): Encyclopedia of Artificial Intelligence. New York: Wiley-Interscience Publication, 285-293.

7. Mares, M. (2000). Fuzzy coalition structures. Fuzzy sets and systems. Elsevier Sciences. 114: 23-33.

8. Nwana, H. S., Ndumu, D. T., Lee, L. C., and Collis, J. C. (1999), ZEUS: A Toolkit for Building Distributed Multi-Agent Systems, Applied Artificial Intelligence Journal 13 (1/2): 129-185.

9. Romero, J. \& Sheremetov, L. (2002). Model of Cooperation in Multi Agent Systems with Fuzzy Coalitions. In B. Dunin-Keplicz, E. Nawarecki (Eds.): From Theory to Practice in Multi-Agent Systems. Lecture Notes in Artificial Intelligence, Springer Verlag, 2296: 263-272.

10. Shehory, O. and Kraus, S. (1999). Feasible Formation of Coalitions Among Autonomous Agents in Non-Super-Additive Environments, Computational Intelligence, Vol. 15(3): 218-251.

11. Smirnov, A. (1999). Virtual Enterprise Configuration Management. In: Proceedings of the 14th IFAC World Congress (IFAC'99), Beijing, China, Pergamon Press, vol. A, 337-342.

12. Smirnov, A. and Chilov, N. (1999). Management Decision Making Models and Tools for Estimating Strategic Objectives of Virtual Enterprise Participants. In: Proceedings of the International Conference on Life Cycle Approaches to Production Systems: Management, Control, Supervision (ASl'99), Leuven, Belgium, 5.10-5.11.

13. Yokoo, M. and Hirayama, K. (2000). Algorithms for Distributed Constraint Satisfaction: A Review. Autonomous Agents and Multi-Agent Systems, Vol. 3(2): 198-212. 